\title{
Insights into Functionality-Specific Adsorption Dynamics and Stable Reaction Intermediates Using Fast Field Cycling NMR
}

J. Ward-Williams ${ }^{\mathrm{a}}$, J-P. Korb ${ }^{\mathrm{b}}$ and L. F. Gladden ${ }^{\mathrm{a}^{*}}$

${ }^{a}$ Department of Chemical Engineering and Biotechnology, University of Cambridge, West Cambridge Site, Philippa Fawcett Drive, Cambridge CB3 0AS, U.K.

b Sorbonne Université, UPMC Univ. Paris 06, CNRS, PHENIX laboratory, F-75005, Paris, France

* Corresponding author: $\underline{\text { fgg1@ cam.ac.uk }}$

13 May 2018

Revised: 3 August 2018 


\begin{abstract}
Fast field cycling, FFC, NMR relaxometry experiments are reported in the frequency range of $10 \mathrm{kHz}-40 \mathrm{MHz}$ to characterise the molecular dynamics of a series of protic (methanol and water) and aprotic (DMSO, acetone, cyclohexane and n-heptane) adsorbates on a $\gamma$-alumina surface of catalytic interest. By analysing the data in the $T_{1}$ domain two distinct peaks were observed for both methanol and acetone. In the case of methanol the two peaks have been shown to originate from the two chemical environments of methanol, which at low field strengths differentiate the $\mathrm{O}^{1} \mathrm{H}$ and alkyl ${ }^{1} \mathrm{H}$ interactions with the surface. In contrast, the second environment of adsorbed acetone is assigned to a stable reaction intermediate formed during an aldol reaction, which strongly influences other molecular adsorption processes at the surface. Inversion of the FFC-NMR data into the $T_{1}$-domain enables the combination of the ability of low field NMR to characterise relaxation modes directly associated with adsorption with $T_{1}$ measurements specific to functional groups and reaction intermediates, thereby avoiding misinterpretation of molecular adsorption characteristics and giving greater insight into adsorption and catalytic behaviour than is possible from the overall relaxation dispersion profiles alone.
\end{abstract}




\section{Introduction}

An understanding of solid-liquid and solid-gas interaction is central to understanding the behaviour of heterogeneous catalysts ${ }^{1}$ and the performance of adsorbents used in separation processes. ${ }^{2}$ In the context of catalysis, tuning of the strength of the relevant solid-fluid interactions can have striking effects on reaction yields and selectivities. ${ }^{3,4}$ As such, the importance of understanding interaction strength has been recognised, and many techniques have been developed and optimised in order to quantify this effect. ${ }^{5-7}$ Heterogeneous systems, however, are highly complex leading to no single technique being universally successful. Often measurements are performed under conditions that do not reflect the true reaction system. For example, temperature programmed desorption, requires the variation of the temperature by several hundreds of degrees and is known to cause decomposition of starting materials and products. ${ }^{8}$ Infrared studies have been highly successful for in situ characterisation, ${ }^{9}$ however these studies tend to be limited to gas phase heterogeneous reactions as many common liquids absorb infrared radiation too effectively to be of widespread use. ${ }^{10,11}$ In contrast, NMR offers an excellent alternative in terms of in situ measurements of interaction strength for adsorbed liquid systems, as it is non-invasive and sensitive to even small changes in the surface behaviour. ${ }^{12}$

This study focusses on the application and interpretation of NMR relaxometry at very low magnetic fields, to probe the dynamics of several adsorbed liquids on a $\gamma$-alumina surface. The longitudinal relaxation time, $T_{1}$, of a spin system is well known to be modulated by molecular motions. ${ }^{13}$ Therefore, the change in $T_{1}$ from the free to the bound state is indicative of the degree of restriction of molecular motions caused by binding - and hence a measure of interaction strength. At very low fields, the relaxation behaviour is controlled by slow surface translational processes and therefore becomes an indirect measure of molecular surface diffusion. ${ }^{14}$ To access the low fields required for such a measurement whilst still retaining a sufficient signal-to-noise ratio, techniques based around rapidly switching magnetic fields i.e. fast field cycling, FFC, NMR are often employed. ${ }^{14,15}$

To date, only a few catalytic systems have been studied using FFC-NMR. The role of coke on the relaxation behaviour of water, DMSO and heptane adsorbed on $\mathrm{Al}_{2} \mathrm{O}_{3}$ and $\mathrm{PtRe} / \mathrm{Al}_{2} \mathrm{O}_{3},{ }^{16}$ water on titania polymorphs, ${ }^{17}$ and water on MCM-41 and SEOS surfaces ${ }^{18}$ have all been explored. Previously our group has also investigated the relative interaction strengths of butanone, 2-propanol and water on a $\mathrm{Pd} / \mathrm{Al}_{2} \mathrm{O}_{3}$ catalyst using FFC-NMR and compared the resulting data with fixed-field measurements of $T_{1} / T_{2}$ as a probe of surface interaction strength. ${ }^{19}$ More generally FFC-NMR has also been applied to study surface interactions in a range of porous media including silica glasses, ${ }^{20-22}$ cement pastes, ${ }^{23,24}$ rock cores,${ }^{25-27}$ and granular packings. ${ }^{28}$

One drawback of FFC-NMR is that it is very difficult to distinguish between different chemical species and concurrent surface processes. In particular, the magnetic field homogeneity is currently too poor to allow chemical shift resolution, and the observed relaxation rates tend to be the average of several simultaneously occurring processes in fast exchange. This leads to monoexponential behaviour being observed in the majority of literature examples. Whilst this simplifies the model fitting procedure, if not analysed carefully an unexpected contribution, such as exchange with the surface hydroxyls, can bias the interpretation of the data. The separation of multiple surface processes through pure 
relaxometry experiments has previously been reported, ${ }^{29}$ however very few examples of this exist for FFC-NMR. Those that do have been attributed to multi modal pore sizes distributions, ${ }^{25}$ a continuous range of pore sizes, ${ }^{30}$ and chemical separation of oil and water binary systems. ${ }^{28}$ Often multicomponent systems are dealt with by taking a logarithmic average of the $T_{1}$ profile, thus once again averaging the complex surface effects. Here, it is demonstrated that minor components can be observed in the $T_{1}$-distributions and nuclear magnetic relaxation dispersion (NMRD) profiles of certain liquids adsorbed onto catalytic surfaces. The origins of these minor components are quite different and reveal important insights into the chemical behaviour of the systems that is not accessible from analysis of the logarithmic average of the $T_{1}$ data.

\section{Materials and methods}

The porous medium used was $\gamma$-alumina (BASF, catalogue number 43855) of pore volume $0.65 \mathrm{ml} \mathrm{g}^{-1}$. Nitrogen adsorption analysis was performed using a Micromeritics TriStar 3000 automated gas adsorption analyser. The pore size characterisation data were then calculated using the Barrett-Joyner-Halenda, BJH, method. The pore size distribution was monomodal and centred around a pore diameter of $10 \mathrm{~nm}$. Electron spin resonance, ESR, measurements were performed on a Bruker E500 X-band spectrometer with an ER 4122SHQE cavity at a microwave frequency of $9.385 \mathrm{GHz}$. Two features were observed for $\gamma$-alumina, a sharp paramagnetic resonance at $1600 \mathrm{G}(6 \mathrm{ppm})$ assigned to $\mathrm{Fe}^{3+}$ and a small broad feature centred about $3450 \mathrm{G}$ which has previously been assigned to superparamagnetic $\mathrm{Fe}^{3+}$ clusters within the framework. ${ }^{31}$ Deionised water was produced in-house using an Elga Purelab DV25 purification system. Cyclohexane, n-heptane, DMSO, acetone and methanol ( $>99 \%$ purity) were obtained from Sigma Aldrich, and mesityl oxide ( $>90 \%$ purity) was obtained from Alfa Aesar. All deuterated variants of methanol were obtained from Fluorochem at a purity $>99 \%$. n-Heptane, DMSO and water adsorbed within alumina have been studied by earlier workers and therefore these data provided a reference point for comparison. ${ }^{16}$ Further, DMSO is polar but less reactive than acetone whilst, of course, having an almost identical molecular structure. Methanol and water are both polar and contain an exchangeable $\mathrm{OH}$ functionality, but with the $\mathrm{OH}$ functionality in methanol being distinct form the $\mathrm{CH}_{3}$ group. This set of liquids therefore provides benchmarking with previous work and the ability to test the robustness and sensitivity of the FFC-NMR measurement to the surface behaviour of these molecules.

Samples were prepared by drying the alumina for $12 \mathrm{~h}$ at $120^{\circ} \mathrm{C}$ to drive off physisorbed water, and then soaking the alumina for a further $12 \mathrm{~h}$ in the desired liquid. The sample was then poured onto filter paper and the extra pellet liquid was removed shortly prior to analysis. Full saturation of the pore space with the liquid during the imbibition process was confirmed gravimetrically by gravimetric measurement of the pellets before and after liquid imbibition.

${ }^{32}$ For degassed samples, three freeze-pump-thaw cycles were performed before flame sealing to minimise the oxygen content of the liquid.

FFC-NMR experiments were performed on a Stelar Spinmaster Duo relaxometer. For each NMRD profile either 20 or $30{ }^{1} \mathrm{H}$ Larmor frequencies were analysed, logarithmically spaced between $10 \mathrm{kHz}$ and $40 \mathrm{MHz}$. A pre-polarized sequence was used for field strengths below 


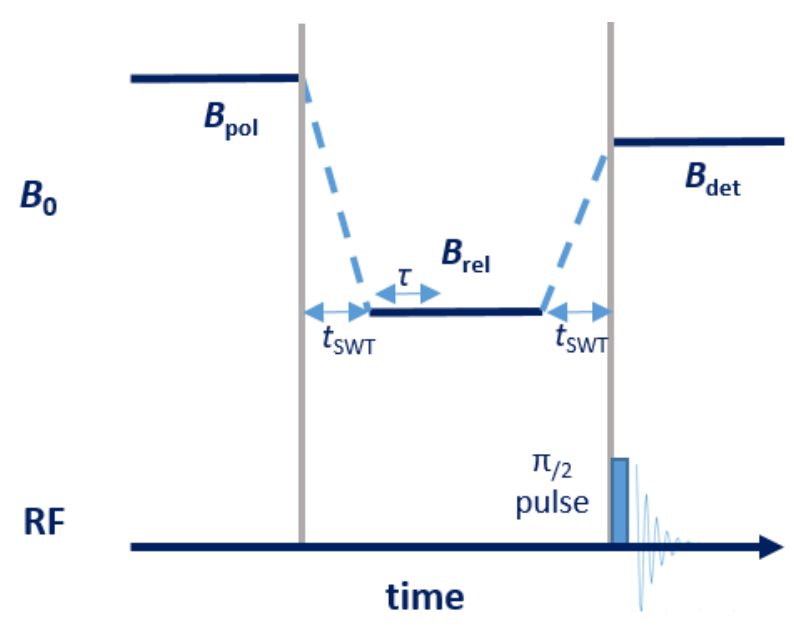

Figure 1: A schematic of the prepolarized FFC-NMR experiment. The spin ensemble is polarized at a field $B_{\text {pol }}$ for $4 \times T_{1}$ before the field is rapidly switched ( $t_{\mathrm{swT}}$ ) to the relaxation field $B_{\text {rel }}$. Relaxation occurs for a single $\tau$ delay before the field is raised to the detection field $B_{\text {det }}$ for acquisition. Repeating this sequence with variable $\tau$ values generates a relaxation profile..$^{14}$

$10 \mathrm{MHz}$ and a non-polarized sequence was used for measurements above $10 \mathrm{MHz}$, as shown schematically in Fig. $1 .^{15}$ The crossover frequency was measured using both sequences to confirm that the two techniques were consistent. For each $T_{1}$ experiment 32 delay times were used and only a single scan was required to achieve a good SNR. The raw FFC-NMR data were renormalized such that the signal in each dataset decayed from 1 to $0 .{ }^{14}$ The $T_{1}$ distribution at each field strength was obtained via an Inverse Laplace transformation using Tikhonov regularisation. ${ }^{33}$ The logarithmic average of the relaxation rate, $\left\langle R_{1}\right\rangle=\left\langle 1 / T_{1}\right\rangle$, was calculated by taking the logarithmic average of the $T_{1}$ distribution at each field strength. ${ }^{30}$ The relative intensity of each peak is extracted directly from the regularisation.

Influences on the quantitative nature of the relative population of the minor peak are as follows. At high field, the $T_{1}$ values of the major and minor peaks become closer (less than a factor of 3 in relaxation time constants) and lead to a systematic bias against the intensity of the minor peak regardless of whether the data are fitted with a multi-exponential or analysed using a regularisation approach. Thus, an under-estimate of the smaller population is obtained. Furthermore, as the relaxation rate of the minor peak approaches the switching time of the FFC-NMR hardware $(3 \mathrm{~ms}$ ) the relative intensity of the minor peak is further reduced in a fashion analogous to the well-known dead-time problem in NMR acquisitions. In the extreme case of methanol, the two peaks are indeed separated by less than a factor of 3 in $T_{1}$ values at frequencies $>1 \mathrm{MHz}$, and the relaxation time of the minor component is small $(\sim 5$ $\mathrm{ms}$ ) at $0.1 \mathrm{MHz}$. Therefore, the reduction in the relative population of the minor peak in $T_{1}$ is particularly noticeable. The errors introduced to the relative peak populations are $\geq 10 \%$ for frequencies $>1 \mathrm{MHz}$, and at frequencies $\leq 0.1 \mathrm{MHz}$ errors in the expected population of the minor species up to $\sim 19 \%$ are observed due to dead-time effects. Limiting the analysis of the relative populations to the range 0.1-1 MHz mitigates these effects with an under-estimate of population of the minor peak being $\sim 9 \%$. For these reasons, when discussing the data reported, the populations assigned to the minor species are taken as average populations in the range $0.1-1 \mathrm{MHz}$. It is noted that the purpose of this paper is not to characterise the populations of the minor species but to identify their existence; however, an upper estimate of the relative populations of the minor species is obtained. Considering the accuracy of the $T_{1}$ 
values obtained, provided that sufficient signal remains to perform the $T_{1}$ measurement then the absolute value of $R_{1}$ is unaffected by the switching time signal loss, but it will still be susceptible to the aforementioned errors at high fields. Typical experimental errors in $\left\langle R_{1}\right\rangle$, calculated from repeat measurements, for single component systems are $<10 \%$, but rise to $15 \%$ for the major component in a two component system and $30 \%$ in the case of the minor component. In both cases the relative populations are associated with an error of $5 \%$.

\section{Results}

Figure 2 shows the logarithmic average of the relaxation profiles, $\left\langle R_{1}\right\rangle=\left\langle 1 / T_{1}\right\rangle$, more commonly referred to as the NMR dispersion curves, obtained for water, methanol, DMSO, acetone, cyclohexane and n-heptane in their bulk liquid state. All 6 liquids showed the same general features of their dispersions (the changes in $\left\langle R_{1}\right\rangle$ ), where $\left\langle R_{1}\right\rangle$ initially increased as the ${ }^{1} \mathrm{H}$ Larmor frequency was decreased, before reaching a plateau around $10 \mathrm{MHz}$. The position of the inflection point was almost identical for all liquids, despite the molecules being significantly different in terms of intermolecular interactions, viscosity, and of unknown dissolved oxygen content. The latter has been previously cited as being responsible for the frequency dispersion seen above $10 \mathrm{MHz} .{ }^{34}$ This was confirmed to be the case in this study by degassing a bulk liquid sample of cyclohexane. The size of the dispersion was reduced from $0.15 \mathrm{~s}^{-1}$ to $0.02 \mathrm{~s}^{-1}$ by degassing, which proved that the small total observable dispersions seen for bulk liquids in Fig. 2 are dominated by the effects of dissolved oxygen rather than any relaxation effects due to the molecular motions of the bulk liquids; hence, for liquids adsorbed on porous media all dispersions seen below $10 \mathrm{MHz}$ can be unambiguously ascribed to interaction of the adsorbate with the surface, even in the presence of dissolved oxygen.

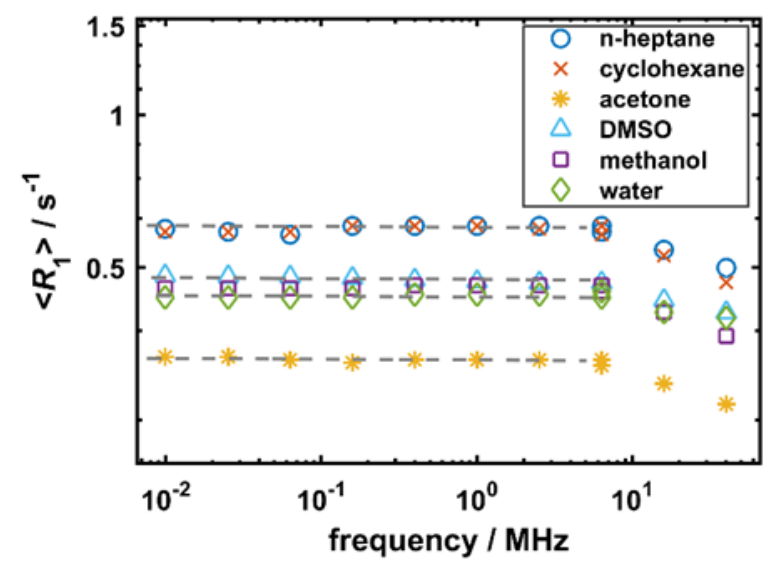

Figure 2: The NMRD profiles of the six different liquids studied in their bulk state. Dashed lines have been included as a guide to the eye for the frequency behaviour below $10 \mathrm{MHz}$.

Figure 3 shows the $\left\langle R_{l}\right\rangle$ profiles for the six different liquids imbibed within $\gamma$-alumina as a function of the ${ }^{1} \mathrm{H}$ Larmor frequency. To avoid any ambiguity in terms of the origins of the relaxation behaviour all further discussions will relate to NMRD profiles below $10 \mathrm{MHz}$, however the full profile will be shown in figures for completeness. As bulk liquids showed no change of $\left\langle R_{1}\right\rangle$ with respect to frequency below $10 \mathrm{MHz}$, the rate of change of $\left\langle R_{1}\right\rangle$ for 


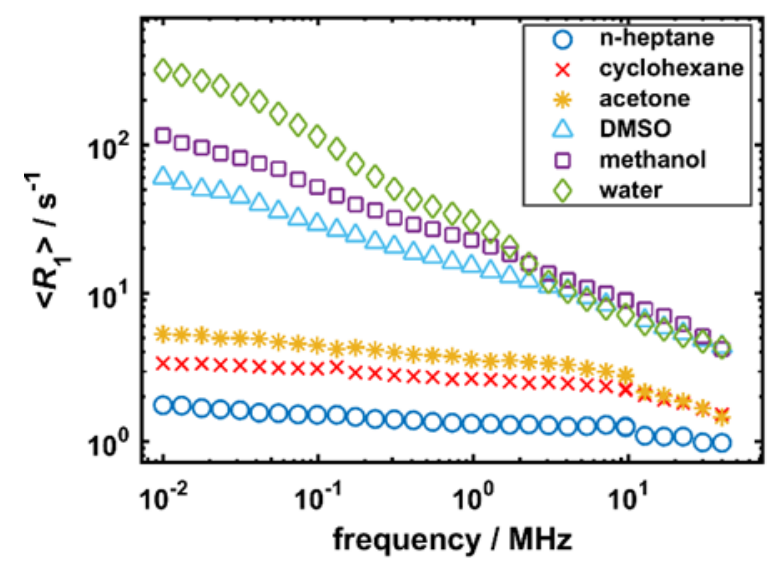

Figure 3: The NMRD profiles of n-heptane, cyclohexane, acetone, DMSO, methanol and water imbibed within porous $\gamma$-alumina pellets.

liquids adsorbed on $\gamma$-alumina is expected to correlate with interaction strength. The total observable relaxation dispersion (TOD), defined as the difference in relaxation rate at $10 \mathrm{kHz}$ and $10 \mathrm{MHz}$, alongside physical parameters of each adsorbate are listed in Table 1.

Qualitatively the interaction strength can be ranked as water > methanol > DMSO > acetone $>$ cyclohexane $\approx$ n-heptane. This is consistent with the order of values of polarity predicted from Reichardt analysis $;{ }^{35}$ these values of polarity are expected to be a reliable indicator of bonding strength for molecules interacting with a polar surface. However, it is noticed that acetone is much more similar to n-heptane and cyclohexane in terms of its dispersion behaviour than DMSO. Interpreting the data qualitatively in terms of an apparent power law allowed a direct comparison to previous studies of water, DMSO and n-heptane on an alumina support. ${ }^{16}$ Power law exponents of $-0.6,-0.35$ and -0.05 respectively were reported ${ }^{16}$, which agree to within experimental error with the values observed in this study $(-0.59,-0.31$ and -0.05$)$.

Table 1: Physical parameters and the total observable dispersion, TOD, for each of the adsorbates imbibed within $\gamma$-alumina.

\begin{tabular}{cccccc}
\hline adsorbate & $\begin{array}{c}\boldsymbol{d} \\
/ \AA^{36}\end{array}$ & $\begin{array}{c}\text { viscosity } \\
/ \mathbf{c P}^{37}\end{array}$ & $\begin{array}{c}\text { Molar } \\
\text { volume / } \\
\mathbf{c m}^{\mathbf{3}} \mathbf{~ m o l}^{-1}\end{array}$ & $\begin{array}{c}\text { relative } \\
\text { polarity }\end{array}$ & $\begin{array}{c}\text { TOD } \\
/ \mathbf{s}^{-1}\end{array}$ \\
\hline water & 3.3 & 0.89 & 18 & 1.00 & 310 \\
methanol & $3.6^{38}$ & 0.60 & 41 & 0.76 & 100 \\
DMSO & $7.1^{39}$ & 2.00 & 71 & 0.44 & 55 \\
acetone & 6.1 & 0.33 & 74 & 0.36 & 2.1 \\
cyclohexane & 6.2 & 0.98 & 108 & 0.01 & 1.2 \\
n-heptane & 5.9 & 0.41 & 147 & 0.01 & 0.6 \\
\hline
\end{tabular}

Transformation of the acquired data into the $T_{1}$ domain for each frequency studied, provides additional insight, as is seen in Fig. 4. While the data for water, DMSO, cyclohexane and 


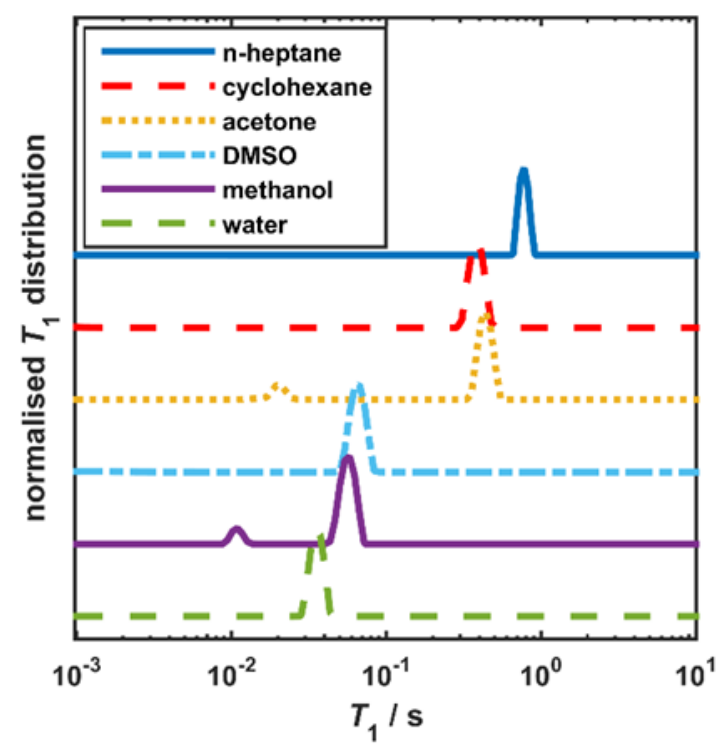

Figure 4: $T_{1}$ distributions of each of the six different liquids adsorbed on $\gamma$-alumina recorded at a frequency of 1 MHz. Data are normalised such that the main peak has a height of one.

n-heptane exhibit single component $T_{1}$ distribution behaviour, consistent with previous relaxation time analyses of these systems ${ }^{16,19,40-42}$, the data for methanol and acetone clearly show two $T_{1}$ components. In both cases this minor component was associated with a $T_{1}$ time constant approximately 1-2 orders of magnitude smaller than the main component. The $T_{1}$ values showed a strong dependence on the Larmor frequency, indicating that the signal was not an artefact. The origins of these minor environments will now be identified and used to improve the understanding of the adsorption process on $\gamma$-alumina.

\section{Discussion}

First, the case of adsorbed methanol is considered. It is proposed that the two environments are associated with the two chemically distinct ${ }^{1} \mathrm{H}$ environments in methanol; this is consistent with the results of high field NMR relaxometry in which the $\mathrm{O}^{1} \mathrm{H}$ and alkyl ${ }^{1} \mathrm{H}$ species are associated with different values of $T_{1}$ when adsorbed onto metal oxide surfaces. ${ }^{29}$ As it was not possible to spectroscopically resolve the peaks using FFC-NMR, a series of partial deuteration experiments were performed to unambiguously assign the two methanol environments. Figure 5 shows the relaxation rate data acquired for $\mathrm{CH}_{3} \mathrm{OH}$ imbibed in $\gamma$ alumina along with samples of partially deuterated methanol $\left(\mathrm{CH}_{3} \mathrm{OD}\right.$ and $\left.\mathrm{CD}_{3} \mathrm{OH}\right)$ imbibed in $\gamma$-alumina. It is seen that the two-component ${ }^{1} \mathrm{H}$ NMRD behaviour of methanol on $\gamma$ alumina is reduced to a single-component decay for both $\mathrm{CD}_{3} \mathrm{OH}$ and $\mathrm{CH}_{3} \mathrm{OD}$. It is clear from a comparison of partially deuterated methanol and non-deuterated methanol that the fast relaxing minor environment must be the hydroxyl protons and the slow relaxing major environment is associated with the alkyl protons. On closer inspection of the data it is seen that the $T_{1}$ values of the partially deuterated molecules deviate slightly from those recorded for the fully protonated methanol sample. This is expected as the relaxation behaviour is 


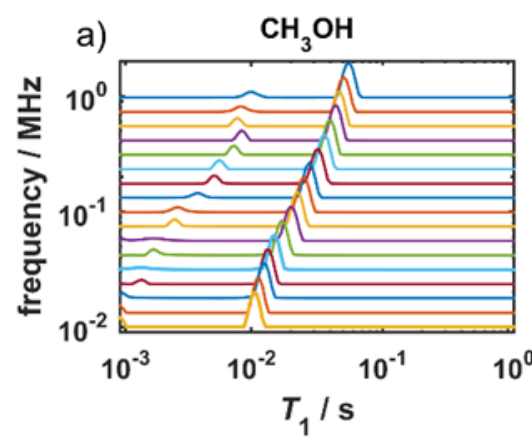

d)

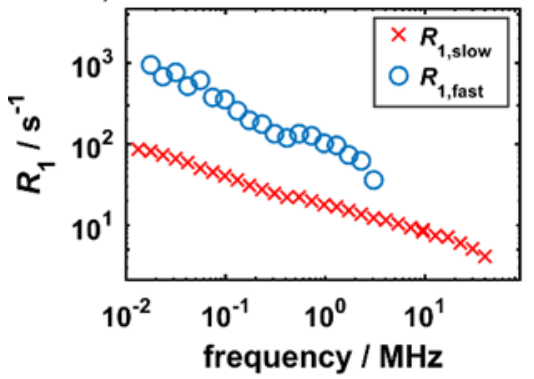

b)

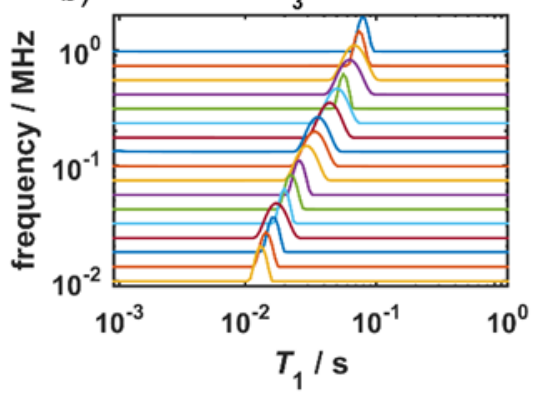

e)

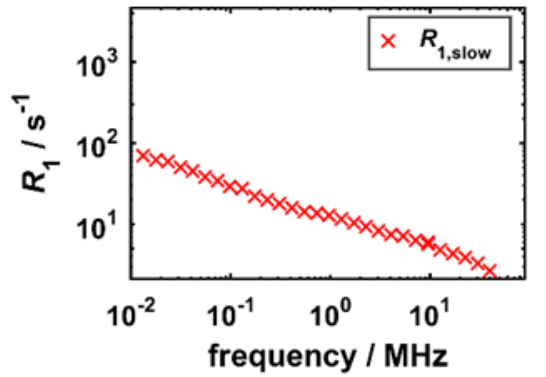

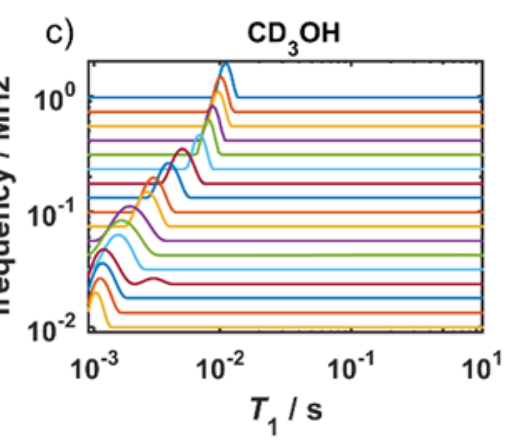

f)

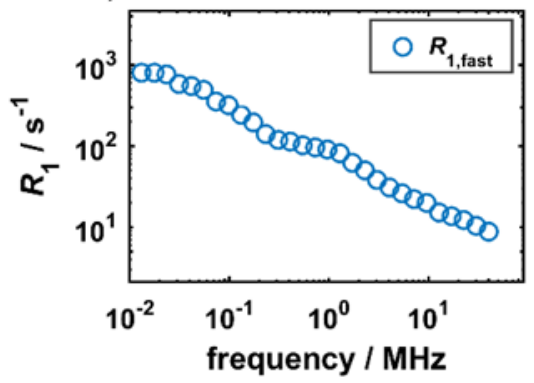

Figure 5: $T_{1}$ distributions, normalised as in Fig. 4, recorded at a frequency value of $\leq 1 \mathrm{MHz}$, and below the corresponding NMRD profile calculated from the modal values of the $T_{1}$ distributions for $\mathrm{CH}_{3} \mathrm{OH}(\mathrm{a}, \mathrm{d}), \mathrm{CH}_{3} \mathrm{OD}$ $(\mathrm{b}, \mathrm{e})$ and $\mathrm{CD}_{3} \mathrm{OH}(\mathrm{c}, \mathrm{f})$. The estimate of the relative population of the minor peak is calculated from data in the frequency range 0.1-1.0 MHz.

complicated by the introduction of ${ }^{1} \mathrm{H}-{ }^{2} \mathrm{H}$ and ${ }^{2} \mathrm{H}-{ }^{2} \mathrm{H}$ couplings, but the effect is small and does not add ambiguity to the assignment of each relaxation environment. Assignment of the peaks to the $\mathrm{O}^{1} \mathrm{H}$ and alkyl ${ }^{1} \mathrm{H}$ environments requires that the hydroxyl group experiences more effective relaxation than the alkyl group. The most likely cause of this enhanced relaxation is the ability of the hydroxyl environment to exchange with the surface. This exchange process leads to the observed $T_{1}$ of the hydroxyl group being a weighted average of the true methanol hydroxyl $T_{1}$ and the surface hydroxyl $T_{1}$ (which is expected to be significantly smaller). This interpretation is confirmed by the observation that the ${ }^{1} \mathrm{H}$ NMRD profile of methanol- $\mathrm{d}_{4}$ adsorbed on $\gamma$-alumina showed the same relaxation profile as methanol- $\mathrm{d}_{3}$. The only source of ${ }^{1} \mathrm{H}$ that could have been incorporated into methanol- $\mathrm{d}_{4}$, and therefore detected by FFC-NMR, must have originated on the catalyst surface and, under fast exchange conditions, been incorporated into the methanol molecule. The relative of the population of the $\mathrm{O}^{1} \mathrm{H}$ peak averaged over the frequency range $0.1-1.0 \mathrm{MHz}$ was $\sim 16 \%$. The population measured from the data is therefore consistent with the expected relative population of $25 \%$ if an under-estimate of $9 \%$ is assumed, as discussed earlier.

Assignment of the two peaks observed in the acetone data, shown in Fig. 6, is now considered. The symmetry of an acetone molecule means that it is not possible to resolve the two methyl groups chemically and thus an alternative explanation of the minor environment is required. The presence of an additional relaxation environment and the acid-base nature of $\gamma$-alumina is consistent with an aldol condensation of acetone on the $\gamma$-alumina surface to form mesityl oxide, and therefore the second species observed could be either mesityl oxide or an aldol intermediate. A schematic of such a reaction scheme is shown in Fig. $7 .^{43}$ 

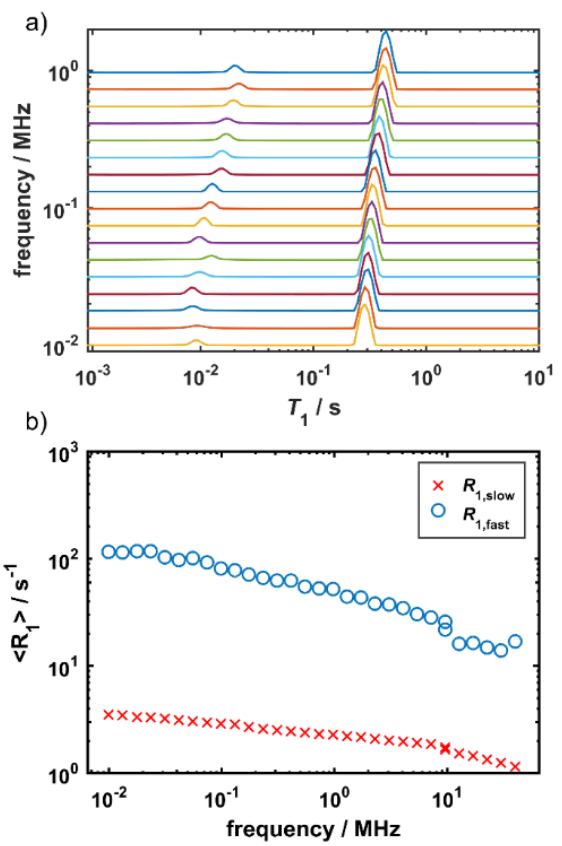

Figure 6: $T_{1}$ distributions, normalised as in Fig. 4, recorded at a frequency value of $\leq 1 \mathrm{MHz}$, and below the corresponding NMRD profile calculated from the modal values of the $T_{1}$ distributions for acetone. The estimate of the relative population of the minor peak is calculated from data in the frequency range $0.1-1.0 \mathrm{MHz}$.

To investigate the nature of this second species, additional FFC-NMR experiments were performed on the same $\gamma$-alumina fully-saturated in single-component mesityl oxide, and a 50:50 mol.\% mixture of mesityl oxide and acetone. The dispersion curves for these two samples compared with the data for single-component acetone in $\gamma$-alumina are shown in Fig. $8 \mathrm{a}$, and the data recorded at $17 \mathrm{kHz}$ were transformed into the $T_{1}$ domain in Fig. 8b. From Fig. $8 \mathrm{a}$ it is seen that the dispersion data for pure acetone and the mixture of acetone and mesityl oxide appear identical to within experimental error, whilst the data for mesityl oxide shows a much larger dispersion, indicating a greater interaction with the surface. This result is surprising, because the aldol condensation of acetone to mesityl oxide is an established heterogeneous catalytic conversion. The apparently much stronger interaction of the product, mesityl oxide, than the reactant, acetone, with the $\gamma$-alumina surface would suggest that the catalyst surface would bind the product strongly, inhibiting further conversion; such behaviour is not typical of this reaction. Further, the similarity of dispersion curves for acetone and the 50:50 mol.\% mixture of mesityl oxide and acetone also suggests that the mesityl oxide is characterised by a much weaker interaction with the surface when coimbibed with acetone. Insight as to the processes that are occurring, as well as a tentative assignment of the second species formed by acetone adsorption is provided by inspecting the 
$\mathbf{A}$

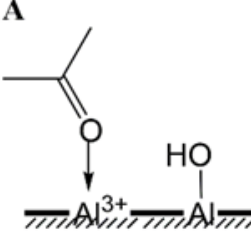

B

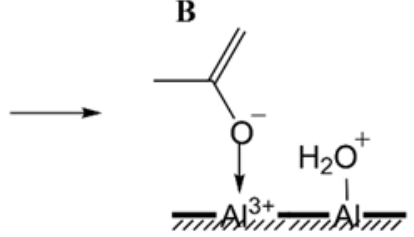

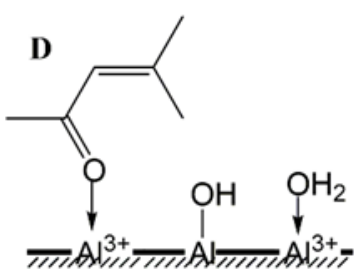

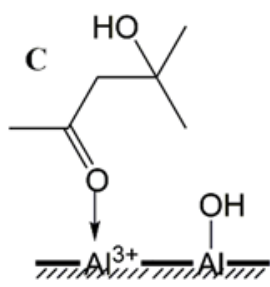

Figure 7: A schematic reaction scheme for the formation of mesityl oxide from acetone over a $\gamma$-alumina surface via an aldol condensation reaction. ${ }^{43}$ The scheme shows A) acetone adsorbed on the hydroxylated alumina surface, B) an enolate intermediate caused by deprotonation of acetone, C) a $\beta$-hydroxy ketone intermediate formed by the attack of the enolate into a second equivalent of acetone and D) the subsequent dehydration product, mesityl oxide. Species B and C can be considered as aldol intermediates, and this aldol reaction can be generalised to all aldehydes and ketones that possess $\alpha$-hydrogens.
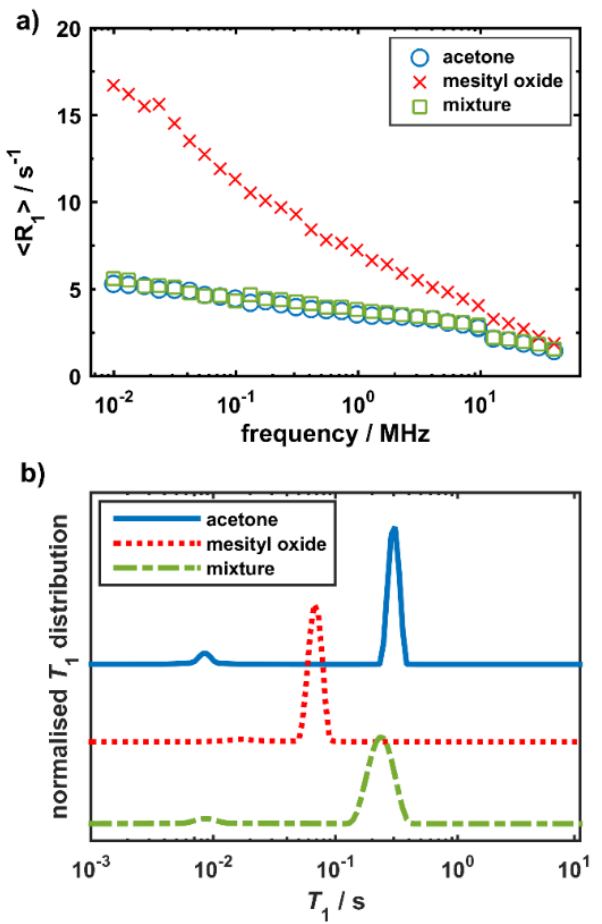

Figure 8: The a) NMRD profile of acetone, mesityl oxide and a 50:50 mol. \% mixture of acetone and mesityl oxide on $\gamma$-alumina and $\mathrm{b}$ ) the $T_{1}$ distributions measured for each of the adsorbates at a ${ }^{1} \mathrm{H}$ Larmor frequency of $17 \mathrm{kHz}$.

data in the $T_{1}$-domain. From Fig. $8 \mathrm{~b}$ it is clearly seen that while the $T_{1}$ of the major components in the $T_{1}$ data does occur at a smaller value of $T_{1}$ for mesityl oxide than for acetone, consistent with the dispersion profiles in Fig. 8a, there is a clearly defined minor peak in the acetone data occurring at about $T_{1}=10^{-2} \mathrm{~s}$. Averaging the $T_{1}$ distribution data 
over the frequency range $0.1-1 \mathrm{MHz}$ showed that $\sim 12 \%$ of the acetone $T_{1}$ population distribution data was associated with this minor peak, which is a stable and strongly binding reaction intermediate of acetone. It follows that a possible explanation of the acetone and mesityl oxide single-component data is that the in the case of acetone the strongest adsorption sites facilitate the aldol condensation reaction, thereby forming a steady-state population of aldol intermediate interacting strongly with the alumina surface. The $85-90 \%$ of the acetone remaining unreacted interacts less strongly with the surface, either interacting with less strong adsorption sites or shielded from the surface by the strongly adsorbing intermediate. It is noted that a small second peak can also be detected in the single-component mesityl oxide $T_{1}$ distribution. The population of this peak does not exceed $5 \%$ (frequency range $0.1-1 \mathrm{MHz}$ ) of the total $T_{1}$ population distribution.

The existence of the minor peak during acetone adsorption strongly suggests that the system is capable of undergoing an aldol condensation reaction and that the observed intermediate in the mesityl oxide data is the analogous enolate or $\beta$-hydroxy ketone intermediate formed via the mechanism shown in Fig. 7. However, the steady-state concentration of the aldol intermediate is lower - likely due to mesityl oxide being a poorer substrate for aldol reactions than acetone. ${ }^{44}$ The remaining $95 \%$ of the signal originates from unreacted mesityl oxide, which has greater access to the surface and strong binding sites compared to the acetone system due to the reduced proportion of strongly adsorbing intermediates - resulting in a much lower value of $T_{1}$. The $T_{1}$ distribution acquired for the 50:50 mol.\% mixture of mesityl oxide and acetone also showed a major and minor peak. Although it is a 50:50 mixture, the $T_{1}$ of the main peak is weighted more towards that of the single component acetone data suggesting that mesityl oxide is much less strongly interacting with the surface in the presence of acetone. A minor peak representing $\sim 10 \%$ of the population (frequency range $0.1-1 \mathrm{MHz}$ ) is also observed which is consistent with the above arguments. By adding the more reactive substrate, acetone, to the mesityl oxide system, the concentration of aldol intermediates is increased, and as a result of the competitive adsorption process the $T_{1}$ of the major peak increases. Under these conditions the $T_{1}$, and hence the expected interaction with the $\gamma$-alumina surface, are very similar for acetone and mesityl oxide, and also essentially identical to that of single component acetone.

Therefore, it is seen that by simply taking the logarithmic average dispersion data for singlecomponent mesityl oxide and acetone the competitive adsorption behaviour of the two species is not predicted. However, by comparing the data for the two single-component systems in the $T_{1}$-domain, the binary adsorption behaviour is not only predicted, but also the evidence for the stable reaction intermediate present during single-component adsorption and the adsorption characteristics of the binary are identified. Therefore, the information gathered from the single component systems is sufficient to predict the behaviour of the binary system; the $T_{1}$ domain analysis is therefore demonstrated to be a rapid and informative method for analysing FFC-NMR data. This approach is not limited to the formation of reaction intermediates and can theoretically be generalised to any form of in situ competitive adsorption processes, such as water promotion effects.

To support this interpretation of the $T_{1}$ domain data, ${ }^{1} \mathrm{H}$ spectroscopy of acetone on $\gamma$-alumina was performed at $300 \mathrm{MHz}$, and showed a peak at approximately $6 \mathrm{ppm}$ with respect to the ${ }^{1} \mathrm{H}$ resonance of tetramethylsilane, indicative of double bond formation (see supporting information). The intensity of the unsaturated bond peak was far too small, however, for the 
minor environment to be mesityl oxide itself, nor was there any conclusive evidence of the coproduct (water) being formed. A surface species has previously been observed at room temperature and pressure through IR studies of acetone on alumina, which was tentatively suggested to be either an enolate or a $\beta$-hydroxy ketone as shown in Fig. $7 .{ }^{43}{ }^{13} \mathrm{C}$ NMR spectroscopy performed at $75 \mathrm{MHz}$ confirmed the presence of mesityl oxide and the $\beta$ hydroxy ketone species, consistent with the observations from the FFC.

Finally, it is now possible to explain the apparently anomalous data shown in Table 1, in which it was noted that the dispersion behaviour (TOD) of acetone is much more similar to nheptane and cyclohexane than DMSO, even though the polarity of DMSO is much closer to that of acetone than to the alkanes. It is now seen that the stable adsorption intermediate in the acetone- $\gamma$-alumina system modifies the adsorption properties of acetone. In this case, the reaction intermediate is more strongly bound to the surface than acetone and prevents the unreacted acetone from interacting significantly with the surface. Thus, the TOD observed for acetone is much smaller than would be expected on the basis of its polarity. In contrast, DMSO, which has almost identical structure and polarity to acetone does not form an intermediate on the $\gamma$-alumina surface and is associated with dispersion behaviour much more expected based on its polarity. Further, the FFC-NMR data give insight as to why acetone is more reactive than DMSO on the $\gamma$-alumina surface. For completeness, it is noted that the TOD of methanol, which also shows two peaks in the $T_{1}$ distribution, is dominated by the major component of the logarithmic average as a result of population weighting effects. Therefore to a good approximation the data only represent the non-exchangeable protons in this system, and methanol demonstrates the expected behaviour in terms of its TOD characteristic and relative polarity in Table 1.

\section{Conclusions}

At frequencies lower than $10 \mathrm{MHz}$ only interactions of the adsorbate with the solid surface give rise to dispersion behaviour. In this range, fast field cycling NMR relaxometry has been used to successfully rank the interaction strength of a series of small molecules imbibed within $\gamma$-alumina. The resolution between the different adsorbates was sufficiently high to allow an ordering of interaction strength, which showed that water $>$ methanol $>$ DMSO > acetone $>$ cyclohexane $\approx$ heptane, which was consistent with the relative polarities of these species. By considering the data in the $T_{1}$ domain, it was clear that two of the six adsorbates (methanol and acetone) showed two component relaxation behaviour, which was characterised in terms of relative magnitude and relaxation rate. Errors influencing the relative peak intensities and the values of $T_{1}$ obtained are discussed.

For methanol the two peaks were unambiguously identified as the hydroxyl $\mathrm{O}^{1} \mathrm{H}$ and alkyl ${ }^{1} \mathrm{H}$ proton environments. The $\mathrm{O}^{1} \mathrm{H}$ relaxation which is influenced by exchange with the $\gamma$ alumina surface hydroxyls, was observed to be up to an order of magnitude faster than the alkyl ${ }^{1} \mathrm{H}$ environment at low frequencies. It follows that where the $\mathrm{O}^{1} \mathrm{H}$ functionality plays a role in the catalytic process it is important that it is the interaction of that $\mathrm{O}^{1} \mathrm{H}$ functionality with the surface that is characterised, and therefore data should be analysed in the $T_{1}$ domain. Analysis of the logarithmic average dispersion will give a characterisation of the interaction increasingly weighted by the ${ }^{1} \mathrm{H}$ species associated with the less-strongly interacting alkyl ${ }^{1} \mathrm{H}$ 
species and may therefore give a misleading or insensitive indication of the affinity of the reactive group for the surface.

Finally, for acetone the observation of a stable reaction intermediate was confirmed through FFC-NMR and the analysis of these data in the $T_{1}$ domain. It was shown that the formation of these aldol intermediates blocks strong surface binding sites and prevents the remaining molecules from interacting effectively with the surface. By considering the relaxation data in the $T_{1}$ domain the distinction between chemical activity and average interaction strength of a molecule with the surface is clear and can be used to predict the behaviour of a binary mixture. Again, this enhanced understanding is not possible through a simple logarithmic average of the $R_{1}$ data. We suggest that this FFC-NMR methodology can be generalised to the understanding of any competitive adsorption process, such as catalyst poisoning and water promotion effects which are of great importance in the field of heterogeneous catalysis. $^{45,46}$

\section{Supporting information}

${ }^{1} \mathrm{H}$ and ${ }^{13} \mathrm{C}$ spectra of acetone adsorbed on $\gamma$-alumina

\section{Acknowledgements}

The authors thank Sam Schott for acquiring the ESR data, and the EPSRC (grant number EP/M507350/1) for financial support. 


\section{References}

(1) Nørskov, J. K.; Bligaard, T.; Rossmeisl, J.; Christensen, C. H. Towards the Computational Design of Solid Catalysts. Nat. Chem. 2009, 1, 37-46.

(2) Li, J.-R.; Kuppler, R. J.; Zhou, H.-C. Selective Gas Adsorption and Separation in Metal - Organic Frameworks. Chem. Soc. Rev. 2009, 38, 1477-1504.

(3) Jenness, G. R.; Schmidt, J. R. Unraveling the Role of Metal-Support Interactions in Heterogeneous Catalysis: Oxygenate Selectivity in Fischer-Tropsch Synthesis. ACS catal. 2013, 3, 2881-2890.

(4) Medford, A. J.; Vojvodic, A.; Hummelshøj, J. S.; Voss, J.; Abild-Pedersen, F.; Studt, F.; Bligaard, T.; Nilsson, A.; Nørskov, J. K. From the Sabatier Principle to a Predictive Theory of Transition-Metal Heterogeneous Catalysis. J. Catal. 2015, 328, 36-42.

(5) Mojet, B. L.; Ebbesen, S. D.; Lefferts, L. Light at the Interface: the Potential of Attenuated Total Reflection Infrared Spectroscopy for Understanding Heterogeneous Catalysis in Water. Chem. Soc. Rev. 2010, 39, 4643-4355.

(6) Barrie, P. J. Analysis of Temperature Programmed Desorption (TPD) Data for the Characterisation of Catalysts Containing a Distribution of Adsorption Sites. Phys. Chem. Chem. Phys. 2008, 10, 1688-1696.

(7) Pan, H.; Ritter, J. A.; Balbuena, P. B. Isosteric Heats of Adsorption on Carbon Predicted by Density Functional Theory. Ind. Eng. Chem. Res. 1998, 37, 1159-1166.

(8) Carrizosa, I.; Munuera, G. Study of the Interaction of Aliphatic Alcohols with $\mathrm{TiO}_{2}$. J. Catal. 1977, 49, 174-188.

(9) Hernández-Giménez, A. M.; Ruiz-Martínez, J.; Puértolas, B.; Pérez-Ramírez, J.; Bruijnincx, P. C. A.; Weckhuysen, B. M. Operando Spectroscopy of the Gas-Phase Aldol Condensation of Propanal over Solid Base Catalysts. Top. Catal. 2017, 60, $1522-1536$.

(10) Andanson, J.-M.; Baiker, A. Exploring Catalytic Solid/Liquid Interfaces by In Situ Attenuated Total Reflection Infrared Spectroscopy. Chem. Soc. Rev 2010, 39, 45714584.

(11) Roy, D.; Liu, S.; Woods, B. L.; Siler, A. R.; Fourkas, J. T. Nonpolar Adsorption at the Silica/Methanol Interface: Surface Mediated Polarity and Solvent Density across a Strongly Associating Solid/Liquid Boundary. J. Phys. Chem. C 2013, 117, 27052-27061.

(12) D’Agostino, C.; Brett, G. L.; Miedziak, P. J.; Knight, D. W.; Hutchings, G. J.; Gladden, L. F.; Mantle, M. D. Understanding the Solvent Effect on the Catalytic Oxidation of 1,4-Butanediol in Methanol over $\mathrm{Au} / \mathrm{TiO}_{2}$ Catalyst: NMR Diffusion and Relaxation Studies. Chem. Eur. J. 2012, 18, 14426-14433.

(13) Abragam, A. Principles of Nuclear Magnetism; Oxford University Press: Oxford, UK, 1983.

(14) Kimmich, R.; Anoardo, E. Field-Cycling NMR Relaxometry. Prog. Nucl. Mag. Res. Sp. 2004, 44, 257-320. 
(15) Ferrante, G.; Sykora, S. Technical Aspects of Fast Field Cycling. Adv. Inorg. Chem. 2005, 57, 405-470.

(16) Stapf, S.; Ren, X.; Talnishnikh, E.; Blümich, B. Spatial Distribution of Coke Residues in Porous Catalyst Pellets Analyzed by Field-Cycling Relaxometry and Parameter Imaging. Magn. Reson. Imaging 2005, 23, 383-386.

(17) Conte, P.; Loddo, V.; De Pasquale, C.; Marsala, V.; Alonzo, G.; Palmisano, L. Nature of Interactions at the Interface of Two Water-Saturated Commercial $\mathrm{TiO}_{2}$ Polymorphs. J. Phys. Chem. C 2013, 117, 5269-5273.

(18) Chemmi, H.; Petit, D.; Levitz, P.; Denoyel, R.; Galarneau, A.; Korb, J.-P. Noninvasive Experimental Evidence of the Linear Pore Size Dependence of Water Diffusion in Nanoconfinement. J. Phys. Chem. Lett. 2016, 7, 393-398.

(19) Mitchell, J.; Broche, L. M.; Chandrasekera, T. C.; Lurie, D. J.; Gladden, L. F. Exploring Surface Interactions in Catalysts Using Low-Field Nuclear Magnetic Resonance. J. Phys. Chem. C 2013, 117, 17699-17706.

(20) Stapf, S.; Kimmich, R.; Seitter, R. O.; Maklakov, A. I.; Skirda, V. D. Proton and Deuteron Field-Cycling NMR Relaxometry of Liquids Confined in Porous Glasses. Phys. Rev. Lett. 1996, 115, 107-114.

(21) Korb, J.-P.; Whaley Hodges, M.; Gobron, T.; Bryant, R. G. Anomalous Surface Diffusion of Water Compared to Aprotic Liquids in Nanopores. Phys. Rev. E 1999, 60, 3097-3106.

(22) Mattea, C.; Kimmich, R.; Ardelean, I.; Wonorahardjo, S.; Farrher, G. Molecular Exchange Dynamics in Partially Filled Microscale and Nanoscale Pores of Silica Glasses Studied by Field-Cycling Nuclear Magnetic Resonance Relaxometry. J. Chem. Phys. 2004, 121, 10648-10656.

(23) Korb, J.-P.; Monteilhet, L.; McDonald, P. J.; Mitchell, J. Microstructure and Texture of Hydrated Cement-Based Materials: A Proton Field Cycling Relaxometry Approach. Cem. Concr. Res. 2007, 37, 295-302.

(24) Faux, D. A.; Mcdonald, P. J. Explicit Calculation of Nuclear-Magnetic-Resonance Relaxation Rates in Small Pores to Elucidate Molecular-Scale Fluid Dynamics. Phys. Rev. E 2017, 95, 1-16.

(25) Korb, J.-P.; Freiman, G.; Nicot, B.; Ligneul, P. Dynamical Surface Affinity of Diphasic Liquids as a Probe of Wettability of Multimodal Porous Media. Phys. Rev. E 2009, 80, 61601 .

(26) Godefroy, S.; Korb, J.-P.; Fleury, M.; Bryant, R. G. Surface Nuclear Magnetic Relaxation and Dynamics of Water and Oil in Macroporous Media. Phys. Rev. E 2001, 64, 1-13.

(27) Korb, J.-P.; Nicot, B.; Bubici, S.; Ferrante, G. Dynamics and Wettability of Oil and Water in Oil Shales. J. Phys. Chem. C 2014, 118, 23212-23218.

(28) Korb, J. P.; Godefroy, S.; Fleury, M. Surface Nuclear Magnetic Relaxation and Dynamics of Water and Oil in Granular Packings and Rocks. Magn. Reson. Imaging 2003, 21, 193-199.

(29) Stubner, B.; Knozinger, H.; Conard, J.; Fripiat J., J. Adsorption of Alcohols on 
Alumina. 2. Nuclear Magnetic Resonance Investigation. J. Chem. Phys. 1978, 82, 1811-1817.

(30) Korb, J.; Ferrante, G.; Bubici, S.; Mallett, M. New Instrumental Platform for the Exploitation of the Field- Dependence of $T_{1}$ in Rock Core Analysis and Petroleum Fluids : Application to $T_{1}-T_{2}$ Correlation Maps. Diffus. Fundementals 2014, 22, 1-7.

(31) Djemai, A.; Balan, E.; Morin, G.; Labbe, J. C.; Pierre, J. Behaviour of Paramagnetic Iron during the Thermal Transformations of Kaolinite. J. Am. Ceram. Soc. 2001, 84, 1017-1024.

(32) Hollewand, M. P.; Gladden, L. F. Transport Heterogeneity in Porous pellets-I. PGSE NMR Studies. Chem. Eng. Sci. 1995, 50, 309-326.

(33) Butler, J. P.; Reeds, J. A.; Dawson, S. J. Estimating Solutions of First Kind Integral Equations with Nonnegative Constraints and Optimal Smoothing. J. Numer. Anal. 1981, 18, 381-397.

(34) Teng, C.; Hong, H.; Kiihne, S.; Bryant, R. G. Molecular Oxygen Spin-Lattice Relaxation in Solutions Measured by Proton Magnetic Relaxation Dispersion. J. Mag. Res 2001, 34, 31-34.

(35) Reichardt, C. Solvents and Solvent Effects in Organic Chemistry; Wiley-VCH Publishers: Weinheim, Germany, 2003.

(36) Webster, C. E.; Drago, R. S.; Zerner, M. C.; Gaines, V. Molecular Dimensions for Adsorptives. J. Am. Chem. Soc 1998, 120, 5509-5516.

(37) Smallwood, I. M. Handbook of Organic Solvent Properties, Arnold: London, UK, 1996.

(38) Wu, H.; Gong, Q.; Olson, D. H.; Li, J. Commensurate Adsorption of Hydrocarbons and Alcohols in Microporous Metal Organic Frameworks. Chem. Rev. 2012, 112, 836868.

(39) Korb, J.-P. Multiscale Nuclear Magnetic Relaxation Dispersion of Complex Liquids in Bulk and Confinement. Prog. Nucl. Mag. Res. Sp. 2018, 104, 12-55.

(40) Korb, J. -P. Nuclear Magnetic Relaxation of Liquids in Porous Media. New J. Phys. 2011, 13, 35016-1-26.

(41) Badea, C., Pop, A., Mattea, C. Stapf, S. Ardelean, I. The Effect of Curing Temperature on Early Hydration. Appl. Magn. Reson. 2014, 45, 1299-1309.

(42) De Pasquale, C.; Marsala, V.; Berns, A. E.; Valagussa, M.; Pozzi, A.; Alonzo, G.; Conte, P. Fast Field Cycling NMR Relaxometry Characterization of Biochars Obtained from an Industrial Thermochemical Process. J. Soils Sediments 2012, 12, 1211-1221.

(43) Zaki, M. I.; Hasan, M. A.; Pasupulety, L. Surface Chemistry of Acetone on Metal Oxides: IR Observation of Acetone Adsorption and Consequent Surface Reactions on Silica-Alumina versus Silica and Alumina. Langmuir 2000, 16, 430-436.

(44) Gamman, J. J.; Jackson, S. D.; Wigzell, F. A. Synthesis of Methyl Isobutyl Ketone over $\mathrm{Pd} / \mathrm{MgO} / \mathrm{SiO}_{2}$. Ind. Eng. Chem. Res. 2010, 49, 8439-8443.

(45) Hutchings, G. J. Promotion in Heterogeneous Catalysis : A Topic Requiring a New Approach? Perspect. Catal. 2001, 75, 1-12. 
(46) Davies, P. R. On the Role of Water in Heterogeneous Catalysis: A Tribute to Professor M. Wyn Roberts. Top. Catal. 2016, 59, 671-677. 


\section{TOC Graphic}

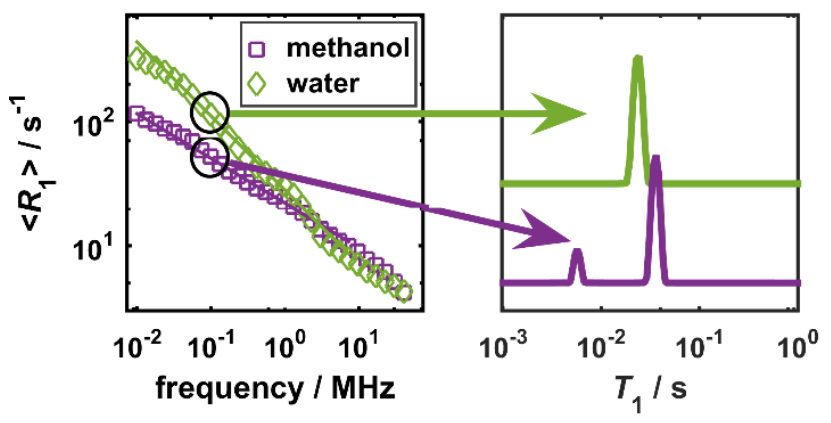

